\title{
Histopathology of Microvascular Anastomosis- Comparison of Patent and Nonpatent Anastomosis: An Experimental Study
}

\author{
Dhaval Gohil ${ }^{1, \odot}$ Nasser Mohammed ${ }^{2, \odot}$ Anita Mahadevan ${ }^{3, \odot} \quad$ Nupur Pruthi ${ }^{1}$ \\ ${ }^{1}$ Department of Neurosurgery, National Institute of Mental Health \\ and Neurosciences, Bangalore, Karnataka, India \\ ${ }^{2}$ Department of Neurosurgery, University of Virginia, \\ Charlottesville, Virginia, United States \\ Address for correspondence Nupur Pruthi, MCh,Department of \\ Neurosurgery, Second Floor Neuro-faculty Block, National Institute \\ of Mental Health and Neurosciences, Bangalore 560029, Karnataka, \\ India (e-mail: pruthi_nupur@yahoo.co.in).
}

${ }^{3}$ Department of Neuropathology, National Institute of Mental

Health and Neurosciences, Bangalore, Karnataka, India

Indian J Neurosurg 2022;11:7-12

\begin{abstract}
Objective To compare the histopathology of patent and nonpatent microvascular anastomosis using rat femoral artery end-to-end anastomosis model.

Materials and Methods In 15 Sprague-Dawley rats, end-to-end anastomosis was performed on the right femoral artery. The classical method was used in four cases and one-way up method in 11 cases. The animals were sacrificed after 2 weeks and the anastomosis was subjected to histopathology. The pathological changes in patent and nonpatent cases were compared.

Results The immediate patency rate and delayed patency (after 2 weeks) rate was $86.7 \%$ and $66.7 \%$, respectively. The mean follow-up was 3 months. At sacrifice, $5 / 15$ anastomosis were not patent. Marked subintimal thickening was noted in $4 / 5$ (80\%) of the nonpatent group, which was absent in the patent group. Severe loss or fibrosis of tunica media and marked adventitial inflammation were noted in all nonpatent cases $(5 / 5,100 \%)$. As much as $4 / 5$ of the nonpatent cases had poor or indeterminate apposition; in contrast, good apposition was seen in 6/10 (60\%) of the patent group. The mean clamp time and mean suturing time were significantly longer in the nonpatent group (69.2 minutes and 53.8 minutes, respectively) as compared with the patent group (48.8 minutes and 31.8 minutes, respectively). A single case that was initially

Keywords

- histopathology

- microvascular anastomosis

- patency nonpatent was found to have recanalized at 6 months.

Conclusion Minimal intimal injury and reaction, minimal thinning of tunica media, mild-to-moderate adventitial changes, good apposition, and equidistant sutures were associated with a successful microvascular anastomosis. Short duration of vessel clamping time and suturing comes with experience and dedicated practice in a skills laboratory.
\end{abstract}

\section{Introduction}

The Cushing's banyan tree has certainly grown over the past 100 years, since he described about the growing subspecialties of medicine in his paper "the special field of neurological surgery" written in $1905 .^{1}$ The special field

published online

March 25, 2021
DOI https://doi.org/

$10.1055 / s-0040-1719237$ ISSN 2277-954X. of cerebrovascular surgery has evolved now into submillimeter vascular anastomosis. Microvascular anastomosis surgery plays a significant role in human central nervous system (CNS) diseases. The major indications include selected patients with ischemic cerebrovascular disease

(C) 2021. Neurological Surgeons' Society of India.

This is an open access article published by Thieme under the terms of the Creative Commons Attribution-NonDerivative-NonCommercial-License, permitting copying and reproduction so long as the original work is given appropriate credit. Contents may not be used for commercial purposes, or adapted, remixed, transformed or built upon. (https://creativecommons.org/licenses/by-nc-nd/4.0/).

Thieme Medical and Scientific Publishers Pvt. Ltd. A-12, 2nd Floor, Sector 2, Noida-201301 UP, India 
like moyamoya disease, complex aneurysms where direct clip reconstruction is not possible, and tumors that warrant vessel sacrifice to achieve complete resection.

Microvascular anastomosis has a steep learning curve., ${ }^{2,3}$ It can be mastered only by dedicated practice in a skills laboratory. Apart from the surgical skill that is required, a successful microvascular anastomosis is a result of intriguing cascade of histopathological processes that are essentially modulated by the surgical intervention and influenced greatly by the technique of the surgeon.

The neurosurgery literature is lacking in the field of microvascular anastomosis, particularly in studying histological perspective of patent and nonpatent anastomosis. The present study is an effort to elucidate factors responsible for a failure of microvascular anastomosis. This study looks at both histopathological changes and technical factors that determine the fate of an anastomosis using an end-to-end anastomosis model in rat femoral artery.

\section{Materials and Methods}

Fifteen Sprague-Dawley rats were used for the study. The study was approved by Institutional Animal Ethics Committee. Procedures were performed under sterile precautions. The details of the procedure are below.

Femoral artery end-to-end anastomosis 4 : after anaesthetizing the rats, an oblique groin incision was made. Femoral vessels were identified. The femoral artery was dissected free from its perivascular sheath. One or more branches joining the proximal portion of femoral artery were defined and ligated and cut to mobilize the artery. The femoral artery was divided between clamps sharply. An amount of $1 \%$ lidocaine is then applied over the artery to relieve vasospasm and achieve vessel dilatation that aids in anastomosis. A colored background was placed below the vessels for better visualization. Nylon 10-0 was used as the suture material. The classical method of suturing was used in four cases and one-way up technique was used in 11 cases. In the classical method, two sutures were placed in the anterior wall approximately 120 degrees apart. The part of the anterior wall was again divided into three equal parts by two equidistant sutures. Then, the clamps were rotated such that the posterior wall was turned anterior. Then, a suture was placed exactly midway between the previous stitches on the back wall. This suture served as a stay suture. This divided the remaining unsutured part of the vessel's circumference into two equal halves. In most, two sutures were paced in each of these halves. This completed the anastomosis with around nine stitches. The "one-way up" technique, also called "posterior wall first" technique, is used in situations when the vessel cannot be turned over. Here, the first stitch is made at 180 degrees away from the surgeon and used as a stay. Then, starting first from the posterior wall, stitches are applied, and the anterior wall is sutured. Once suturing was completed, the clamps were released and a cotton gauze or fat pad was used to gently tamponade the anastomotic leakage which eventually stopped in all cases. The immediate patency was checked using the empty and refill test proposed by Hayhurst and O'Brien in $1975^{5}$ : A pair of forceps is used to grasp one end of the anastomosis and using another pair of forceps, the vessel is emptied by milking out and released to check for refill. The parameters like suturing time, number of sutures used, the clamping time, and immediate patency were studied.

Following the anastomosis, the wound was closed under aseptic precautions. The animals were reversed from anesthesia and observed in postoperative room for 1 day and later shifted to their cages. There were no cases of limb ischemia or gangrene; this could be explained, in part, due to effective collateral blood supply of the rat hind limbs. The wound was re-explored at a later date (mean 3 months) under general anesthesia, and the anastomotic site was examined for delayed patency. The artery was then harvested and subjected to histopathological analysis.

\section{Histopathological Analysis}

The resected segment of artery with anastomotic site was fixed in $10 \%$ neutral buffered formalin for 2 to 3 weeks prior to processing for paraffin embedding. The arterial segment was embedded in transverse and longitudinal planes. Serial 3- to 4-micron thick sections were cut and stained with hematoxylin-eosin (H\&E), and special stains including Masson's trichome (MAT) and Verhoeff Van Gieson (VVG) were used. MAT is a three-stain technique used to stain collagen in connective tissue (blue color) along with nuclei (dark brown to black) and cytoplasm (pink color). VVG stain is used to stain elastic fibers. The Verhoeff stain attaches to the elastic fibers and the von Gieson stain counter stains the other tissue components. The elastic fibers and cell nuclei are stained black, collagen fibers are stained red, and other tissue elements including cytoplasm are stained yellow. The histopathological sections were then carefully studied, and the following parameters analyzed: endothelial integrity, intimal hyperplasia, medial fibrosis and adventitial reaction (fibrosis, inflammation, giant cell response) for each sample. Each parameter was semiquantitatively recorded as mild $(+)$, moderate $(++)$ and severe $(+++)(-$ Fig. 1$)$.

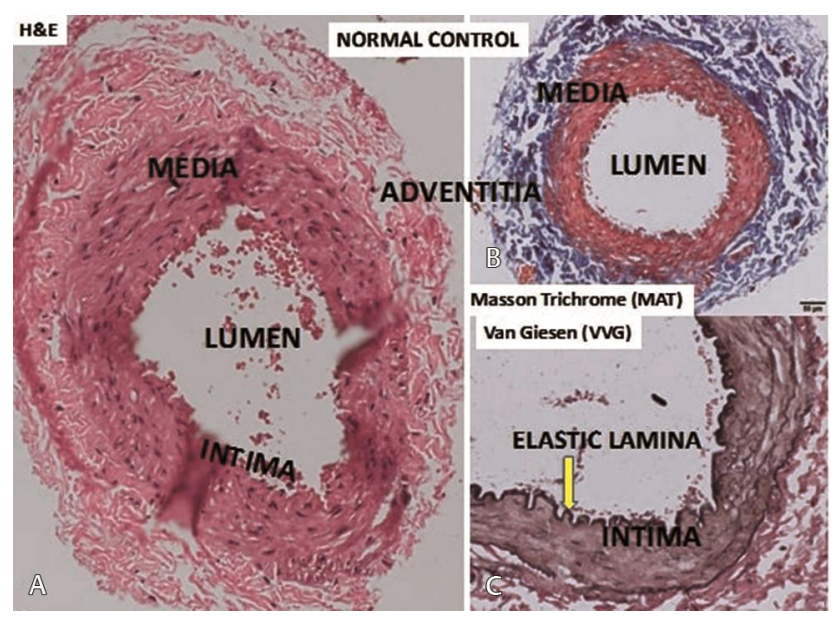

Fig. 1 (A-C): Panel of stains used to study components of vessel. (a) Hematoxylin \& Eosin (H\&E) stain shows lumen enclosed by intima, tunica media and outer tunica adventitia. (B) Masson trichrome (MAT) stain differentially labels tunica media orange and tunica adventitia a deep blue. (C) Verhoff's Van Gieson stain (VVG) labels internal elastic lamina. A: H\&ExObj.1.4; B: MAT stainxObj.1.4; C: VVG stain. 


\section{Results}

\section{Patency Rates}

The immediate patency rate was $13 / 15$ (86.7\%) and delayed patency rate was $10 / 15$ (66.7\%). The mean follow-up period was 3 months (25-120 days). At sacrifice, five were noted to be nonpatent at 3 months postanastomosis. Of these, three were noted to have been "patent" and two "not patent" immediately postsurgery.

\section{Intimal Changes (Fibrosis)}

Mild-to-moderate intimal fibrosis was seen in all $10(100 \%)$ of patent cases and one in the nonpatent group. In contrast, severe intimal fibrosis was seen only in the nonpatent group (4/5 [80\%]).

\section{Changes in Tunica Media}

Changes in tunica media were minimal/only focal in 10/10 $(100 \%)$ of patent cases. Al cases in nonpatent group reveled severe changes with medial thinning/fibrosis (5/5 [100\%]) (-Fig. 2).

\section{Adventitial Inflammation}

Mild-to-moderate adventitial inflammation was seen in $5 / 10$ (50\%) of patent cases. All nonpatent cases revealed severe adventitial inflammation with giant cell response to the suture. However, severe adventitial inflammation was seen in 5/10 (50\%) of patent cases as well.

\section{Apposition}

Good apposition was demonstrable in six (60\%) patent cases. Four out of 5 nonpatent cases had poor or indeterminate apposition.

\section{Distance Between Sutures}

Equidistant sutures were seen in five (50\%) of the patent anastomosis group, which was lacking in all of the nonpatent anastomosis group.

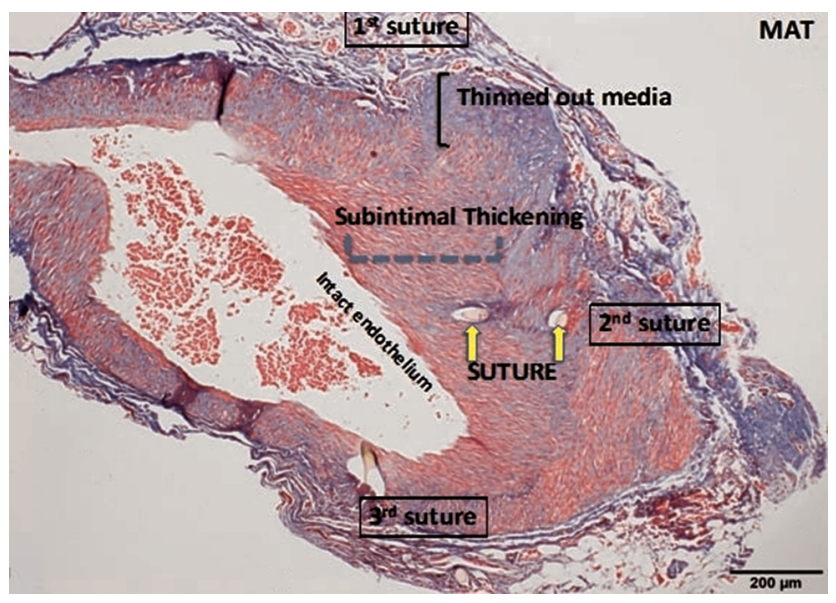

Fig. 2 Stitch through media, causing irregular subintimal fibrosis, with thinning out of media. Lumen is patent with intact endothelium and fairly equidistant sutures. Masson trichrome (MAT) stainxObj.4, magnification $=$ scale bar .

\section{Follow-up and surgery-related parameters}

The clamp time and suturing time were higher in the nonpatent group as compared with the patent group. Adventitial preparation time was longer in the patent group ( - Table $\mathbf{1}$ ).

\section{Discussion}

The success of any microvascular anastomosis is a complex interplay between technique and the resultant cascade of histopathological processes that will ultimately dictate the patency of the anastomosis. Microvascular anastomosis leads to unavoidable vessel injury. Each step of microvascular anastomosis like vessel preparation, transection, clipping, handling, and suturing leads to microvascular injury, which is inevitable. The histopathological changes occurring after the microvascular anastomosis can be studied sequentially, as described below.

There is tissue necrosis within the loop of suture due to the constricting effect of the suture and resultant hyaline degeneration. ${ }^{6}$ A repair reaction is seen around this region. The factors affecting patency of microvascular anastomosis are exhaustive and can be broadly classified according to Chow into technical, local and general factors. ${ }^{6}$ Technical factors include the surgeon, the surgeon's skill, microscope, instruments, suturing technique, suturing time, number of sutures, and suture revision. The local factors include vessel size, intimal damage, medial fibrosis, adventitial reaction, clamping time, vasospasm, tension, and infection. The general factors include temperature, blood pressure, and coagulopathy. The list is exhaustive. The present study aims to elucidate some of the technical and histopathological factors that determine the outcome of microvascular anastomosis.

Endothelial Integrity and Intimal Loss: Endothelial integrity is important for prevention of early vessel occlusion following microvascular anastomosis. Damage to endothelium exposes the subendothelial tissue to blood, causing the initiation of coagulation cascade and culminating in the formation of thrombus, which will occlude the vessel lumen. To ascertain the endothelial integrity, first, the internal elastic lamina is identified. Endothelium is identified by the presence of nuclei above the internal elastic lamina. ${ }^{6}$ Normally, the endothelium is seen as one- or two-layered. The presence of one or two layers of nuclei in one high-power field

Table 1 Follow-up and surgery-related parameters

\begin{tabular}{|l|l|l|}
\hline Factor & Patent $(\boldsymbol{n}=\mathbf{1 0})$ & Nonpatent $(\boldsymbol{n}=\mathbf{5})$ \\
\hline $\begin{array}{l}\text { Follow up } \\
\text { duration (mean) }\end{array}$ & 2.7 months & 3.6 months \\
\hline $\begin{array}{l}\text { Clamp } \\
\text { duration (mean) }\end{array}$ & 48.8 minutes & 69.2 minutes \\
\hline $\begin{array}{l}\text { Suturing } \\
\text { time (mean) }\end{array}$ & 31.8 minutes & 53.8 minutes \\
\hline $\begin{array}{l}\text { Adventitial } \\
\text { preparation (mean) }\end{array}$ & 17 minutes & 15.4 minutes \\
\hline $\begin{array}{l}\text { Number of } \\
\text { sutures (mean) }\end{array}$ & 7 sutures & 8.2 sutures \\
\hline Suture revision & $2(20 \%)$ & $3(60 \%)$ \\
\hline
\end{tabular}


is taken as a criterion for endothelial integrity. ${ }^{6}$ One of the main things to be kept in mind while performing microvascular anastomosis is suture byte should be through and through all three layers of blood vessel rather than mere adventitial or medial suture. The suture through all three layers will maintain continuity (i.e., good apposition) at the anastomosis site. Among all three layers, good intimal apposition is a must for a patent microvascular anastomosis. This is because blood stream will immediately form thrombus if it is exposed to media or adventitial. Hence, suture byte through all three layers of blood vessel and appropriate approximation of intima at anastomosis site is vital for an anastomosis to be patent. Intimal loss is a characteristic feature of early postoperative period. ${ }^{7}$ The causes of intimal damage are mainly related to the dissection technique. Intimal loss can occur during the vessel handling by forceps, vessel dilatation, irrigation, and during stripping of adventitia. Intimal damage is an early event that can lead to loss of patency by promoting thrombus formation, causing vessel occlusion. Careful handling of the vessel end, keeping the endothelial integrity in mind, is the first step toward a successful anastomosis. By the end of second week, endothelial healing is usually complete. ${ }^{8}$

Intimal hyperplasia is a universal response to vessel injury that occurs in a delayed manner, which is especially more common in veins. Damage to endothelium causes vascular smooth muscle cell proliferation. Intimal hyperplasia consists of migrated smooth muscle cells which deposit extracellular matrix and extracellular matrix remodeling. ${ }^{9}$ The break in internal elastic lamina due to vessel trauma during anastomosis and inflammatory cascade causes the myocytes of tunica media to migrate into intima and proliferate, with deposition of collagen causing intimal thickening. Intimal hyperplasia is an important cause of delayed occlusion of the microvascular anastomosis. ${ }^{9}$

In our study, it was observed that severe intimal changes were noted in $4 / 15$ (80\%) of the cases. The nonpatent cases demonstrated a loss of intimal integrity, an occluded and organized thrombus with internal elastic lamina being thrown into folds. All the patent anastomosis (10/15; 100\%) showed milder degree of intimal reaction, which was in the form of intimal hyperplasia, mild subintimal thickening, or mild intimal fibrosis. It is interesting to note that in all patent cases, the intimal integrity was restored. The endothelium was fully repaired and it covered over the anastomotic site. In one case, as demonstrated in the histopathology slide, endothelium had grown and covered a stitch which was exposed to the lumen. The intima showed varying reaction from fibrosis to hyperplasia. Thus, for a patent anastomosis, it can be concluded that endothelial integrity is an important factor determining outcome of microvascular anastomosis.

Medial Fibrosis and Necrosis: Medial necrosis is characterized by loss of nuclei of the smooth muscle cells, thinning and fibrinoid necrosis of the tunica media. ${ }^{6}$ Medial necrosis is more common in thrombosed vessels. In the study by Chow et al, ${ }^{6}$ medial necrosis was seen in $33 \%$ of thrombosed vessels as compared with $16 \%$ of patent anastomosis. In the thrombosed vessels, the media is cut off from the blood stream and medial necrosis invariably occurs. ${ }^{6}$

Our study showed a severe loss or fibrosis of tunica media in all nonpatent cases $(5 / 5 ; 100 \%)$. In contrast, there was minimal or focal thinning of media in all the patent cases (10/10; 100\%). MAT is a three-stain technique used to stain collagen in connective tissue (blue color) along with nuclei and cytoplasm (pink color). This technique demonstrated an increase in connective tissue collagen in tunica media, following microvascular anastomosis consequent to loss of smooth muscle cells, which defines medial fibrosis. Another stain used in our study was VVG stain, which stains elastic fibers black and collagen red in color. Normally, the elastic fibers lamellae are arranged in between muscle cell in the tunica media of muscular arteries. In the present study, the nonpatent cases showed an increase in the elastic fiber content in subintimal zone, and the internal elastic lamina was thrown into large folds. Severe loss of muscle coat of tunica media and medial fibrosis were seen in $100 \%$ (5/5) of nonpatent anastomosis and only 50\% (5/10) of patent anastomosis.

Adventitial Reaction: The adventitial reaction changes were characterized by inflammatory granulation tissue, giant cell response to the suture, and fibrosis outside the external elastic lamina. ${ }^{6}$ The adventitia is usually stripped from the vessel before anastomosis. This is done to prevent intraluminal intrusion of the adventitia, which is strongly thrombogenic. The extent of adventitial reaction is related to the applied dissection technique. ${ }^{10}$ Blunt dissection is more traumatic to adventitia than sharp dissection. ${ }^{10}$ Adventitial reaction is seen in almost all cases. ${ }^{6}$ Chow noted adventitial reaction in $80 \%$ of nonpatent and $90 \%$ of patent anastomosis. Adventitial layer was reported to be thickened with newly formed vasa vasorum. Foreign body giant cell reaction is seen around the sutures. ${ }^{6}$

Other Factors: In our study, equidistance between sutures was seen in 5/10 (50\%) of patent anastomosis. In contrast, none of the cases with nonpatent anastomosis had equidistant sutures. Therefore, equidistant sutures appear to be a critical factor for achieving good outcomes in microvascular anastomosis. The optimum number of sutures for microvascular anastomosis is still debated. Less number of stitches would mean less vessel trauma; however, more number of stitches would achieve better homeostasis. ${ }^{11}$ The ideal number of sutures for microvascular anastomosis varies from 8 to 14 in different published studies. ${ }^{11-13}$ The mean number of sutures in our study was seven in patent group compared with mean of 8.2 sutures in the nonpatent group. Good apposition of vessel wall, as determined by histopathology, was seen in $6 / 10(60 \%)$ of patent group compared with only $1 / 5(20 \%)$ of nonpatent cases. Clamp duration was longer in nonpatent cases (mean 69.2 minutes) as compared with 48.8 minutes in patent group. The mean suturing time was also longer in nonpatent cases (53.8 minutes) as compared with patent cases (31.8 minutes) ( $p$-0.04). Suture revision was performed more often in nonpatent group than patent group. Interestingly, the mean time required for adventitial preparation was more in patent cases (mean 17 minutes) compared with nonpatent cases (mean 15.4 minutes). The adventitial preparation 
requires vessel handling and is believed to predispose to intimal damage. Longer time for adventitial preparation in patent cases may reflect more careful vessel handling. It can be concluded from this study that equidistant sutures, short clamping time, low suturing time, optimal number of sutures, and careful vessel handling during adventitial preparation will yield more favorable results in microvascular anastomosis. Number of knots and knot configuration also plays a significant role in patency rates of microvascular anastomosis. ${ }^{2,3}$ In previous studies, authors have described the superiority of two throw reef knot over the traditional three throw knot in microvascular anastomosis., ${ }^{2,3}$

Possibility for Recanalization: In one of the cases, which was nonpatent, immediate postanastomosis, following sacrifice at 6 months when the anastomosis was studied, was surprisingly noted to have recanalized. The lumen revealed multiple lumina within the organized thrombus in the lumen. The tunica media was thinned out with internal elastic lamina, focally disrupted. Collagen had surrounded each of the small lumina, which was replete with newly formed internal elastic lamina, and lined by endothelium, suggesting that each of the microlumina were functioning within the parent lumen, successfully recanalizing it. However well-formed tunica media was lacking. Hence, even in nonpatent cases, there is a possibility of recanalization at long-term which needs to be kept in mind. The factors that have promoted recanalization need to be further investigated ( $\mathbf{- F i g . 3}$ ).

Microsurgery is generally interpreted as an optical supplement applying the axiom "the better we see, the more we know." However Professor Yasargil said the opposite "the more we know, the more we see." The success of microanastomosis is a complex interplay of several factors. Some of the factors have been analyzed in the
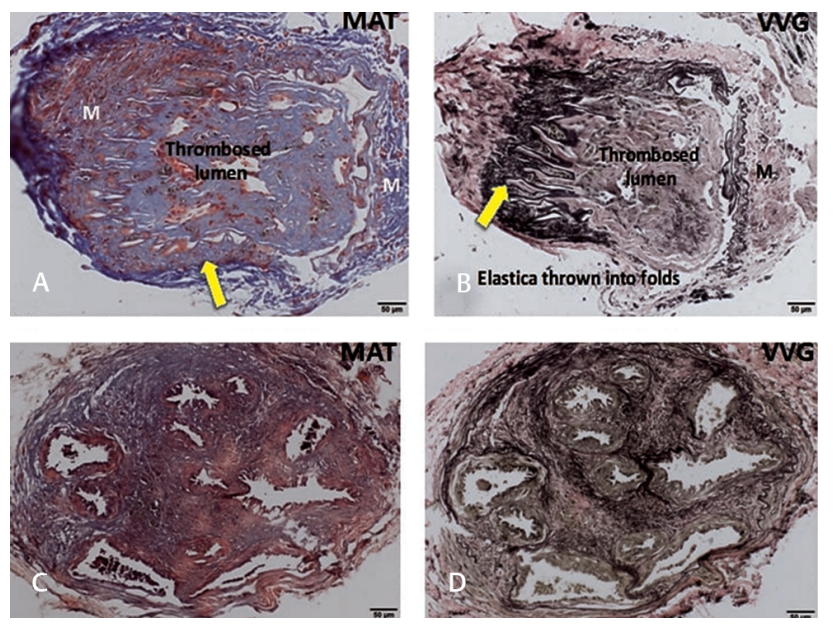

Fig. 3 (A, B): Poor anastomosis causing luminal thrombosis. Note focally thinned out media (A, yellow arrow) and internal elastic lamina thrown into folds (B, yellow arrow). (C, D): Chronic stage (6 months) postanastomosis shows lumen completely recanalized with multiple smaller lumina (C). A thin tunica media and internal elastic lamina is seen around each of the lumina (D). Masson trichrome (MAT) stain, Verhoeff Van Gieson (VVG) stain; magnification = scale bar. present study. There is a significant literature on histology of microvascular anastomosis; $6^{7}$ however, analysis from neurosurgeons is lacking. The present study is a beginning in exploring the pathophysiology of microanastomosis with potential application to treatment of cerebrovascular conditions.

\section{Conclusion}

Successful microvascular anastomosis follows minimal intimal injury and reaction, minimal thinning of tunica media, mild-to-moderate adventitial changes, good apposition, and equidistant sutures. Intimal integrity has to be kept in mind during vessel handling. A reduction in vessel clamping time and suturing time comes with experience and dedicated practice in a skills laboratory, which will positively influence good outcomes. Using the least number of sutures required to balance good apposition and homeostasis is key to favorable outcomes. The possibility of recanalization will also have to be kept in mind.

\section{Conflict of Interest \\ None declared.}

\section{Acknowledgments}

The authors would like to acknowledge the support of the entire staff at Central Animal Research Facility, NIMHANS, Bangalore, India, especially Mr. Ramachandran R (retired senior animal technician) for this work. The authors also acknowledge Mr. Shivaji Rao, senior technician, Department of Neuropathology, for his contribution in slide preparation.

\section{References}

1 Cushing H. The special field of neurological surgery. Bull Johns Hopkins Hosp 1905;16:77-87

2 Pruthi N, Gohil D, Somanna S. Inverted (buried) reef knot in microsurgery - A technical note. Turk Neurosurg 2019;29(4):615-619

3 Nupur P, Dhaval G, Sampath S. Microvascular anastomosis using only 2-throw reef knots: a technical note. Turk Neurosurg 2019;29(6):961-963

4 Pruthi N, Sarma P, Pandey P. Training in micro-vascular anastomosis using rat femoral vessels: comparison of immediate and delayed patency rates. Turk Neurosurg 2018;28(1):56-61

5 Hayhurst JW, O'Brien BM. An experimental study of microvascular technique, patency rates and related factors. Br J Plast Surg 1975;28(2):128-132

6 Chow SP. The histopathology of microvascular anastomosis: a study of the incidence of various tissue changes. Microsurgery 1983;4(1):5-9

7 Acland RD, Trachtenberg L. The histopathology of small arteries following experimental microvascular anastomosis. Plast Reconstr Surg 1977;60(6):868-875

8 Romansky R. Christova: structural change in rat femoral wall after micro surgical anastomosis. Dokl Bulg Akad Nauk 2004;57(12):123-126

9 Lemson MS, Tordoir JH, Daemen MJ A P, Kitslaar PJEHM. Intimal hyperplasia in vascular grafts. Eur J Vasc Endovasc Surg 2000;19(4):336-350 
12 Histopathology of Microvascular Anastomosis Gohil et al.

10 Lohman R, Siemionow M, Lister G. Advantages of sharp adventitial dissection for microvascular anastomoses. Ann Plast Surg 1998;40(6):577-585

11 Wang S, Marini CP, Baso S, Maughan RE, Cunningham JN, Jacobitz IJ. Optimal number of sutures for microvascular anastomosis. Microsurgery 1992;13:161
12 Colen LB, Gonzales FP, Buncke HJ. The relationship between the number of sutures and the strength of microvascular anastomoses. Plast Reconstr Surg 1979;64(3):325-329

13 Robertson JH, Robertson JT. The relationship between suture number and quality of anastomoses in microvascular procedures. Surg Neurol 1978;10(4):241-245 